\title{
Análise eletromiográfica da influência do recrutamento muscular do fibular longo no arco longitudinal comparando o biofeedback e a eletroestimulação neuromuscular Electromyographic analysis of influence of the muscular recruitment of the long fibular in the longitudinal arch comparing the biofeedback and the electrical neuromuscular stimulation
}

Santiago Cattin Munho, Ft. ${ }^{*}$ Fabio Navarro Cyrillo**, Camila Torriani**

${ }^{*}$ Centro Universitário das Faculdades Metropolitanas Unidas - UniFMU ${ }^{* *}$ Professor da UniFMU

\begin{abstract}
Resumo
Objetivo: Este trabalho teve como objetivo verificar a influência do músculo fibular longo no arco longitudinal, e identificar qual dos dois recursos é mais eficaz para o recrutamento muscular: o biofeedback ou a eletroestimulação neuromuscular. Métodos: Seis indivíduos foram selecionados e submetidos a uma avaliaçáo dos arcos plantares no plantígrafo. Para a avaliação da atividade muscular do fibular longo foi utilizada a eletromiografia de superfície. Além destas avaliaçóes, foi realizada a medida da distância do primeiro metatarso e do arco plantar até o solo. De forma randomizada, os indivíduos foram divididos em dois grupos: um grupo fez o recrutamento através do biofeedback eletromiográfico e o outro realizou a eletroestimulação neuromuscular. Após 10 sessóes, foi realizada uma reavaliação no plantígrafo, no eletromiógrafo e das distâncias do primeiro metatarso e do arco plantar até o solo. Resultados: apesar de os dados não terem significância estatística, os resultados sugerem que o biofeedback é mais efetivo no recrutamento do fibular longo que a EENM e que este músculo influencia no aumento do arco plantar. Conclusão: os resultados observados sugerem que para a amostra estudada, o músculo fibular longo tem ação na elevação do arco plantar e que o tratamento com biofeedback eletromiográfico terapêutico foi mais efetivo comparado com a EENM.
\end{abstract}

Palavras-chave: eletromiografia, arco plantar, tratamento, postura.

\begin{abstract}
Objective: The objective of this work is to verify the influence of the long fibular muscle in the longitudinal arch and to identify which method, either neuromuscular electrical stimulation (NMES) or biofeedback therapy, is more effective for the muscle recruitment. Methods: Six subjects were selected and submitted to a plantar arch assessment in the "plantigraphyc". To evaluate long fibular muscle activity, it was used the surface electromyography. The distance of the first metatarsal and the plantar arch until the ground was also evaluated. The individuals were divided into two randomized groups: one made the recruitment with electromyography biofeedback and the other with NMES. At the end of 10 sessions, it was performed another evaluation in the "plantigraphyc", with the electromyography and the distances of first metatarsal and the plantar arch until the ground were taken. Results: Despite the samples did not have statistical importance, the results suggest that the biofeedback is more effective in the recruitment of long fibular muscle than neuromuscular electric stimulation and also that muscle has influence in the plantar arc increase. Conclusion: The results suggest that, for the samples studied, the long fibular muscle elevates the plantar arch and the treatment with electromyography biofeedback is more effective recruiting this muscle than the neuromuscular electric stimulation.
\end{abstract}

Key-words: electromyography, plantar arch, treatment, posture. 


\section{I ntrodução}

O pé é uma estrutura consideravelmente pequena, quando comparada com o quadril, porém, bem mais complexa. Composto por 26 ossos e inúmeros ligamentos dispostos no compartimento medial, lateral, anterior e posterior, destacam-se o talo fibular e o deltóide, que ligam o pé à fíbula e a tíbia e limitam os movimentos de inversão e eversão, respectivamente [1,2].

As articulaçóes do pé envolvem os ossos tarsais, metatarsais e as falanges. As articulaçóes intertársicas mais importantes são a articulação transversa do tarso (articulaçóes calcaneocubóidea e taloclacaneonavicular) e a articulação talocalcânea (subtalar). Além disto, 12 músculos intrínsecos e outros 11 agem sobre o pé $[3,4]$.

O plano de movimento do tornozelo é sagital, possibilitando os movimentos simples de flexão plantar e flexão dorsal [5,2].

A articulaçáo que permite os movimentos de eversão e inversão é a calcâneotalar, com medida passiva entre 4 e 5 graus, em indivíduos jovens para a eversão, sendo que o excesso desse movimento pode deixar o pé valgo $[1,3,4]$.

Os arcos plantares são formados pelos ossos do tarso e metatarsos, no qual 2 deles, os arcos longitudinal medial e lateral, estendem-se desde o calcâneo até os metatarsos e os ossos do tarso. $\mathrm{O}$ arco transverso é formado pelas bases dos metatarsos.

O pé plano ou pé valgo é caracterizado pelo desabamento do arco plantar [6,5]. Esse desabamento pode ocasionar alteraçóes importantes, pois a descarga de peso no pé se altera e muda os eixos das articulaçôes, ocasionando desequilíbrios articulares e musculares em todo o membro inferior, o que pode gerar compensaçóes no joelho e posteriormente no quadril [7].

$\mathrm{O}$ biofeedback pode ser definido como uma retroalimentação de informaçôes. $\mathrm{O}$ Biofeedback eletromiográfico utiliza um equipamento para revelar ou informar ao usuário, de forma visual e/ou auditiva os eventos fisiológicos que ocorrem, durante a contração muscular, possibilitando o controle e modificação destes eventos [8,9]

A atividade muscular, dados goniométricos, eletrocardiograma, temperatura dérmica, freqüência cardíaca são exemplos de eventos fisiológicos que podem ser monitorados e usados terapeuticamente [10].

O biofeedback pode ser usado para o paciente estimular e recrutar um maior número de fibras de um músculo, ou para relaxar um músculo hiperativo [10-12].

O biofeedback é mais comumente usado para o tratamento de incontinência urinaria, porém, não há muitos trabalhos com o uso do recurso no tratamento de problemas posturais como escoliose, pé plano ou cavo [11].

O objetivo deste trabalho foi verificar se o músculo fibular longo apresenta influência no arco longitudinal do pé, identificando qual recurso entre o biofeedback eletromiográfico e a corrente elétrica possibilitam melhor recrutamento do músculo.

A literatura descreve que o desabamento do pé pode trazer conseqüências agravantes para todo o membro inferior, e por este motivo é de suma importância saber quais músculos podem ser recrutados para conter o desabamento deste arco e aumentar sua curvatura. Alguns laboratórios de marcha utilizam o fibular longo para trabalho com arcos desabados.

\section{Materiais e métodos}

Este trabalho foi realizado no laboratório de biofeedback da clínica de fisioterapia do FMU. Seis indivíduos foram selecionados segundo os critérios de exclusão (não poderiam apresentar história de fratura no pé e nem apresentar frouxidão ligamentar). Os sujeitos foram submetidos a uma avaliaçáo postural em simetógrafo e avaliaçáo dos arcos plantares no plantígrafo. Para avaliação da atividade muscular do fibular longo, foi utilizada a eletromiografia de superfície com o aparelho da marca Miotec ${ }^{\oplus}$ modelo Miotool 400 de 4 canais. Foi feita assepsia da regiáo e colocação do eletrodo, segundo Seniam, que era circular, pré-geldado, de $\mathrm{Ag} / \mathrm{ClAg}$ da marca Meditrac.

Além desta avaliação, foi realizada a medida da distância do primeiro metatarso e do arco plantar até o solo.

Os indivíduos foram divididos, aleatoriamente, em dois grupos: um fez o recrutamento por meio de biofeedback eletromiográfico, com séries de dez repetições, dez segundos de contração e dez segundos de repouso; e o outro grupo realizou a eletroestimulação neuromuscular com o aparelho da marca Quark, com freqüência de $80 \mathrm{~Hz}$, em um período de sustentação de dez segundos e repouso de dez segundos, em uma serie de dez repetiçóes.

Os dois grupos realizaram o recrutamento por cinco semanas, duas vezes por semana, com os mesmo parâmetros, totalizando um total de dez sessóes cada.

Ao término das dez sessóes foi realizada uma reavaliação nos mesmos parâmetros anteriores.

\section{Resultados}

Foi utilizada uma técnica não paramétrica de medidas repetidas para dados ordinais. Esta abordagem permite que sejam realizadas as duas comparaçóes citadas anteriormente. Foram verificadas também a existência de interação nos resultados, ou seja, comportamentos diferenciados em função de uma outra variável. Desta forma, a interação pode indicar que os grupos apresentam comportamentos diferenciados de acordo com o momento observado, e pode também indicar que há diferenças entre os tempos para determinado(s) grupo(s), porém para outro(s) não há. Para todas as comparaçôes, foram consideradas haver diferenças quando o resultado do teste apresentou nível descritivo abaixo de 0,05 (p-valor < 0,05), ou seja, o nível de significância adotado foi de $5 \%$.

Por se tratar de variáveis quantitativas, nas descritivas foram utilizadas médias e medianas, para resumir as informaçôes, e desvios-padrão, mínimo e máximo para indicar a variabilidade dos dados. 
Tabela I - Descritivas para as médias dos grupos.

\begin{tabular}{l|l|l|l}
\hline \multicolumn{3}{c}{ Grupo } \\
\cline { 3 - 4 } \multicolumn{1}{l|}{} & Média & 44,3 & 49,8 \\
\cline { 2 - 4 } Média inicial & Mediana & 40,9 & 49,4 \\
& Desvio Padrão & 22,7 & 1,1 \\
& Mínimo & 23,4 & 49,0 \\
& Máximo & 68,5 & 51,1 \\
\hline \multirow{4}{*}{ Média final } & Média & 58,5 & 50,0 \\
& Mediana & 59,0 & 51,7 \\
& Desvio Padrão & 1,1 & 3,3 \\
& Mínimo & 57,2 & 46,2 \\
& Máximo & 59,3 & 52,0 \\
\hline
\end{tabular}

Efeito de interação: $p$-valor $=0,337$

Diferenças entre os momentos (início e reavaliação): $p$-valor $=0,150$

Diferenças entre os grupos: $p$-valor $=0,273$

Por meio dos resultados acima foi possível notar que não houve diferenças entre os momentos ( $p>0,05)$, nem entre os grupos $(\mathrm{p}>0,05)$, porém há fortes evidências de que as diferenças não tenham sido encontradas devido ao baixo tamanho de amostra.

Observando as estatísticas descritivas, observou-se que para o grupo que utilizou o Biofeedback os valores substancialmente aumentaram mais quando comparados com a estimulação neuromuscular.

Tabela II - Descritivas para os valores máximos nos Grupos (primeiro modo).

\begin{tabular}{l|l|l|l}
\hline \multicolumn{2}{c}{ Grupo } \\
\cline { 3 - 4 } \multicolumn{1}{l|}{} & Média & 158,2 & 195,3 \\
Valor máximo & Mediana & 141,0 & 187,0 \\
inicial & Desvio Padrão & 109,2 & 62,9 \\
& Mínimo & 58,6 & 137,0 \\
& Máximo & 275,0 & 262,0 \\
\hline \multirow{4}{*}{ Valor máximo } & Média & 156,2 & 192,7 \\
final & Mediana & 221,0 & 198,0 \\
& Desvio Padrão & 113,2 & 74,1 \\
& Mínimo & 25,5 & 116,0 \\
& Máximo & 222,0 & 264,0 \\
\hline
\end{tabular}

Efeito de interação: $p$-valor $=0,784$

Diferenças entre os momentos (início e reavaliação): $p$-valor $=1,000$

Diferenças entre os grupos: $p$-valor $=0,836$

Observando as estatísticas descritivas, foi possível observar que o grupo que utilizou o Biofeedback apresentou valores máximos maiores do que o grupo EENM em qualquer um dos momentos.
Tabela III - Descritivas para os valores máximos nos Grupos (segundo modo).

\begin{tabular}{l|ll|l}
\hline \multicolumn{3}{l}{} & Grupo \\
\cline { 3 - 4 } \multicolumn{1}{l}{ Valor máximo } & Biofeedback & EENM \\
\hline \multirow{2}{*}{ inicial } & Média & 158,2 & 195,3 \\
& Mediana & 141,0 & 187,0 \\
& Desvio Padrão & 109,2 & 62,9 \\
& Mínimo & 58,6 & 137,0 \\
& Máximo & 275,0 & 262,0 \\
\hline \multirow{4}{*}{ Valor máximo final } & Média & 173,0 & 192,7 \\
& Mediana & 222,0 & 198,0 \\
& Desvio Padrão & 130,2 & 74,1 \\
& Mínimo & 25,5 & 116,0 \\
& Máximo & 271,6 & 264,0 \\
\hline
\end{tabular}

Efeito de interação: $p$-valor $=1,000$

Diferenças entre os momentos (início e reavaliação): $p$-valor $=0,655$

Diferenças entre os grupos: $p$-valor $=1,000$

Pelas descritivas, notou-se que para o grupo que utilizou o Biofeedback, em geral, os valores máximos foram maiores do que o grupo EENM em qualquer um dos momentos. Há indicações também que para o grupo Biofeedback, em média, os valores máximos foram maiores na reavaliação do que no início.

Tabela IV - Descritivas para os desvios padróes dos grupos.

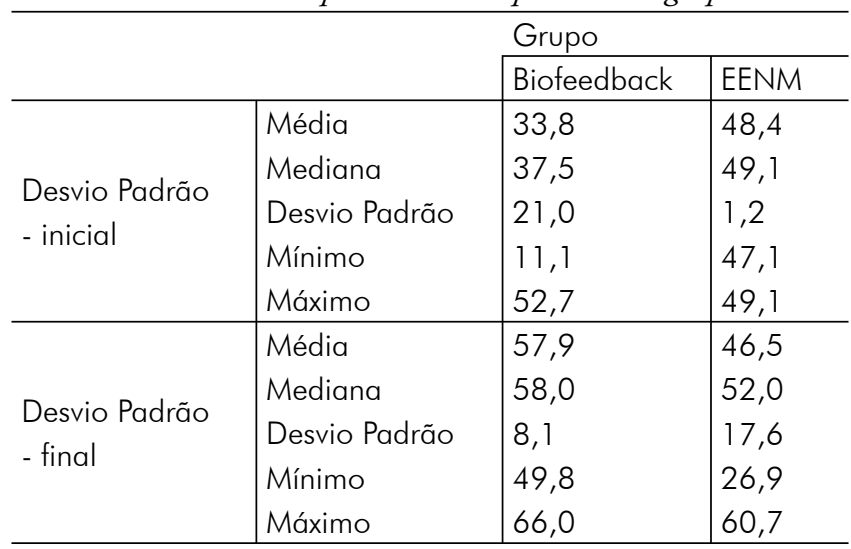

Efeito de interação: $p$-valor $=0,451$

Diferenças entre os momentos (início e reavaliação): $p$-valor $=0,097$

Diferenças entre os grupos: $p$-valor $=0,542$

Para a comparação das diferenças das medidas arco solo, também devido ao baixo tamanho de amostra, foi utilizado o teste não paramétrico de Mann-Whitney. Não foi possível observar diferenças significativas $(\mathrm{p}>0,05)$, porém verificou-se fortes evidências de que o grupo com Biofeedback apresentou maior aumento do início para o final do estudo, e além disto os valores encontrados para o grupo que usou EENM, as diferenças foram bastante baixas. 
Tabela V - Médias de início e reavaliação encontradas entre os grupos.

\begin{tabular}{l|ll|l}
\hline \multicolumn{3}{c}{ Grupo } \\
\cline { 3 - 4 } \multicolumn{2}{c}{} & Biofeedback & EENM \\
\hline \multirow{4}{*}{ Média inicial } & 14,23 & 0,13 \\
& Média & 16,30 & 2,30 \\
& Desvio Padrão & 22,77 & 4,37 \\
& Mínimo & $-9,50$ & $-4,90$ \\
& Máximo & 35,90 & 3,00 \\
\hline
\end{tabular}

p-valor $=0,513$

Tabela VI - Da distância da cabeça do $1^{\circ}$ metatarso e do arco até o solo.

\begin{tabular}{|l|l|l|l|l|}
\hline \multicolumn{4}{|l|}{ Grupo Biofeedback } \\
\hline \multicolumn{4}{|l|}{ Antes } & \multicolumn{2}{l|}{ Depois } \\
\hline Indivíduo 1 & $0,4 \mathrm{~cm}$ & $1,6 \mathrm{~cm}$ & $0,6 \mathrm{~cm}$ & $1,8 \mathrm{~cm}$ \\
\hline Indivíduo 2 & $0,4 \mathrm{~cm}$ & $1,3 \mathrm{~cm}$ & $0,5 \mathrm{~cm}$ & $1,4 \mathrm{~cm}$ \\
\hline Indivíduo 3 & $0,3 \mathrm{~cm}$ & $1,8 \mathrm{~cm}$ & $0,4 \mathrm{~cm}$ & $2,0 \mathrm{~cm}$ \\
\hline Média & $0,36 \mathrm{~cm}$ & $1,56 \mathrm{~cm}$ & $0,5 \mathrm{~cm}$ & $1,73 \mathrm{~cm}$ \\
\hline
\end{tabular}

Tabela VII - Da distância da cabeça do $1^{\circ}$ metatarso e do arco até $o$ solo.

\begin{tabular}{|l|l|l|l|l|}
\hline Grupo EENM & \multicolumn{3}{l|}{ Depois } \\
\hline \multicolumn{4}{|l|}{ Antes } \\
\hline Indivíduo 1 & $0,3 \mathrm{~cm}$ & $1,3 \mathrm{~cm}$ & $0,4 \mathrm{~cm}$ & $1,3 \mathrm{~cm}$ \\
\hline Indivíduo 2 & $0,7 \mathrm{~cm}$ & $2,0 \mathrm{~cm}$ & $0,6 \mathrm{~cm}$ & $2,0 \mathrm{~cm}$ \\
\hline Indivíduo 3 & $0,4 \mathrm{~cm}$ & $1,6 \mathrm{~cm}$ & $0,4 \mathrm{~cm}$ & $1,7 \mathrm{~cm}$ \\
\hline Média & $0,46 \mathrm{~cm}$ & $1,66 \mathrm{~cm}$ & $0,46 \mathrm{~cm}$ & $1,7 \mathrm{~cm}$ \\
\hline
\end{tabular}

\section{Discussão}

A partir dos resultados observados, o estudo sugere que o músculo fibular longo parece auxiliar no aumento do arco longitudinal, mesmo sabendo que ele não é o principal músculo responsável por esta atividade [13].

Kapandji [13] descreve que existem quatro músculos responsáveis pelo aumento do arco plantar, o tibial posterior que é o principal auxiliado pelo fibular longo, flexor do hálux e adutor do hálux e que estes músculos são os responsáveis pela sustentação prolongada do arco plantar. Já Bienfait [14] segue uma linha de raciocínio contraria, dizendo que o arco plantar é sustentado pela aponeurose plantar e seus ligamentos e que os músculos apenas amortecem as pressóes impostas ao arco. Ambos concordam que o músculo tibial posterior é um músculo que age diretamente na elevação do arco plantar, contudo Bienfait acredita que somente os músculos quadrado plantar, flexor curto dos dedos e flexor curto do hálux auxiliam o tibial posterior.

Seguindo Kapandji, este trabalho mostrou que mesmo o fibular longo, não sendo o principal músculo a elevar o arco plantar, é um músculo atuante neste fundamento [13].

O músculo tibial posterior não pôde ser recrutado, neste trabalho, por ser um músculo profundo e de difícil captação de atividade pela eletromiografia de superfície.
Alguns trabalhos como os de Basmanjian, Colborne e Intiso [15-17] mostram que a utilização do biofeedback terapêutico no músculo fibular longo é efetivo para pacientes que apresentam o pé caído decorrente de lesôes neurológicas.

Apesar do número de indivíduos não ter sido suficiente para verificar-se significância estatística, pode-se observar que, em todo momento, o biofeedback terapêutico mostrou-se mais efetivo no recrutamento muscular quando comparado à EENM, tanto para aumento da atividade muscular quanto para o aumento do arco plantar.

Considerando a média de cada grupo, o grupo que realizou o trabalho com biofeedback antes do tratamento tinha a distância de $0,36 \mathrm{~cm}$ da cabeça do primeiro metatarso ao solo e $1,56 \mathrm{~cm}$ do meio do arco plantar ao solo, após o trabalho de recrutamento muscular essa distância passou a ser em média de $0,5 \mathrm{~cm}$ na primeira medida e $1,73 \mathrm{~cm}$ na segunda. Enquanto o grupo que fez o recrutamento com EENM, antes do tratamento apresentava em média uma distância de $0,46 \mathrm{~cm}$ da cabeça do primeiro metatarso ao solo e $1,66 \mathrm{~cm}$ do meio do arco plantar ao solo, e após o trabalho de recrutamento manteve-se a média de $0,46 \mathrm{~cm}$ na primeira medida e $1,7 \mathrm{~cm}$ na segunda medida.

Também observou-se um aumento na distância ente o $1^{\circ}$ metatarso ao solo, sendo que a medida na média, antes do tratamento com Biofeedback, era de $0,36 \mathrm{~cm}$ e após passou a ser de $0,5 \mathrm{~cm}$, um ganho de $0,14 \mathrm{~cm}$. E a medida média do grupo que se tratou com EENM, inicialmente, era de $0,43 \mathrm{~cm}$ passando a ser de $0,5 \mathrm{~cm}$ após tratamento, havendo um ganho de $0,07 \mathrm{~cm}$. Esse ganho pode ser notado quando foi feita uma nova avaliação no plantígrafo, mostrando que a parte medial do pé não tocava tanto o solo quanto na $1^{\text {a }}$ avaliação.

Seria importante fazer uma nova media, seis meses após esse tratamento, para verificar se o aumento do arco foi definitivo ou se apenas temporário, podendo, assim, afirmar se a musculatura auxilia ou não na sustentação do arco plantar como acredita Kapanji.

\section{Conclusão}

Embora o número de amostras não tenha sido suficiente para validação estatística, os resultados observados sugerem que, para a amostra estudada, o músculo fibular longo possui ação na elevaçáo do arco plantar e que o tratamento com biofeedback eletromiográfico terapêutico pode ser mais efetivo no recrutamento muscular do que a EENM.

Vale lembrar que a continuação deste trabalho com um número maior de indivíduos e com um tempo mais prolongado de recrutamento se mostra importante para a confirmaçáo da hipótese levantada.

\section{Referências}

1. Calais-Germain B. Anatomia para o movimento. Barueri: Manole; 1992. 
2. Sobotta J. Atlas de anatomia humana. Rio de Janeiro: Guanabara Koogan; 2000.

3. Moore KL. Anatomia orientada para clínica. Rio de Janeiro: Guanabara Koogan; 2001.

4. Gabrielli C. Arco plantar superficial en el hombre: características anatómicas y comparadas. Rev Chil Anat 1999;17(2):225-30.

5. Hall SJ. Biomecânica básica. Rio de Janeiro: Guanabara Koogan; 2000.

6. Hamill J. Bases biomecânicas do movimento humano. Barueri: Manole; 1999.

7. Bricot B. Posturologia. São Paulo: Ícone; 2001.

8. Robinson AJ, Snyder-Macker L. Eletrofisiologia clínica: eletroterapia e teste eletrofisiológico. Porto Alegre: Artemed; 2001. p.383-397.

9. Durson N, Durson E. Electromyographic biofeedback-controlled exercise versus conservative care for patellofemoral pain syndrome. Arch Phys Med Rehabil 2001; 82(12):1692-5.

10. Soares AV. Biofeedback eletromiográfico na recuperação do pé caído. Rev Fisioter Univ São Paulo 1998;5(2):104-10.
11. Thimothy AH. Eletroterapia clínica. Barueri: Manole; 2003.

12. Cyrillo FN, Torriani C. Biofeedback: conceitos básicos e aplicabilidade clínica. Rev Fisioter UniFMU 2003;1(1):118.

13. Kapandji AI. Fisiologia articular membros inferiores. Rio de Janeiro: Guanabara Koogan; 2000.

14. Bienfait M. Os desequilíbrios estáticos. São Paulo: Summus; 1995.

15. Basmajian JV et al. Biofeedback treatment of foot: drop after stroke compared with standard rehabilitation technique: effect on voluntary control and strength. Arch Phys Med Rehabil 1975;56: 231-6.

16. Colborne GR, Olney SJ, Griffin MP. Feedback of ankle joint and soleus electromyography in the rehabilitation of hemiplegic gait. Arch Phys Med Rehabil 1993;74(1):1100-16.

17. Intiso D, Santilli V, Grasso MG, Rossi R, Caruso I. Rehabilitation of walking with electromyographic biofeedback in footdrop after stroke. Stroke 1994;25(6):1189-92. 